\title{
To pace or not to pace? Or how to pace?
}

\author{
loana Șuș ${ }^{*}, 1,2$ Roxana Rudzik², Dan Dobreanu ${ }^{1,2}$ \\ ${ }^{1}$ University of Medicine and Pharmacy of Tîrgu Mureș, Romania, ${ }^{2}$ Emergency Institute for \\ Cardiovascular Diseases and Transplantation Tîrgu Mureș, Romania
}

\begin{abstract}
Chronic apical right ventricular pacing may impair left ventricular function and cause heart failure in patients with indication for antibradycardia pacing and normal left ventricular ejection fraction at baseline, through multiple electro-mechanical changes. We describe the case of a patient who needed an upgrade to cardiac resynchronization therapy and developed angina early after single chamber right ventricular pacing and discuss pacing induced cardiomyopathy.
\end{abstract}

Keywords: cardiac resynchronization therapy, apical right ventricular pacing, upgrade, heart failure

\section{Introduction}

Right ventricular pacing is preferred in patients with preserved left ventricular function who need antibradycardia pacing due to a lower complication rate and longer service life over biventricular systems. However, in a significant proportion of patients, it may cause pacing induced cardiomyopathy. This has previously been described as a decrease of more de $10 \%$ in left ventricular ejection fraction and heart failure symptoms, for which intraventricular and interventricular dyssynchrony is the main cause. In Pacing to Avoid Cardiac Enlargement trial [1] designed to compare biventricular pacing and right ventricular pacing with regards to left ventricular function in patients with standard indications for pacing, 8 of 9 patients in which the left ventricular ejection fraction decreased to less than $45 \%$ after 12 months of pacing were in the right ventricular pacing group.

Received: January 2017; Accepted after review: April 2017; Published: June 2017.

${ }^{*}$ Corresponding author: loana Șuș, MD, PhD, Cardiology Department, Emergency Institute for Cardiovascular Diseases and Transplantation Tîrgu Mures, Romania, 50, Gheorghe Marinescu Street, 540139, Tîrgu Mureș, Romania

E-mail: susioana@yahoo.com

\section{Case report}

A 75 year-old Caucasian female patient, with history of moderate arterial hypertension, elective angioplasty with drug eluting stent on anterior descending artery and permanent single chamber VVI pacemaker implanted for atrial fibrillation with slow ventricular response and symptomatic ventricular pauses 4 months earlier, was admitted in the cardiology department for progressive exertional dyspnea and repeated episodes of typical angina pectoris. At the moment of pacemaker implantation, the documented echocardiography described a non-dilated left ventricle, with mild concentric hypertrophy and preserved ejection fraction (60\%), and mild mitral regurgitation. The patient was on treatment with beta-blocker (bisoprolol 2.5 $\mathrm{mg}$ ), angiotensin converting enzyme inhibitor (ramipril $2.5 \mathrm{mg}$ ), loop diuretic (furosemide 40 $\mathrm{mg}$ ), calcium channel blocker (amlodipine 5 $\mathrm{mg}$ ) and oral anticoagulant (apixaban $2.5 \mathrm{mg}$ b.i.d).

On physical examination, the patient presented with moderate peripheral edema and bibasilar crackles, heart rate 60 beats-perminute, blood pressure $150 / 90 \mathrm{mmHg}$, systolic murmur grade III/VI in mitral and Botkin-Erb areas. Laboratory tests revealed high serum 
urea and creatinin levels $(100 \mathrm{mg} / \mathrm{dl}, 1.9 \mathrm{mg} / \mathrm{dl}$ respectively), normal troponin I and CK-MB, and high $\mathrm{N}$-terminal pro-brain natriuretic peptide levels, $22012 \mathrm{pg} / \mathrm{ml}$. On ECG, there was atrial fibrillation with paced ventricular rhythm with left bundle branch block morphology (Figure 1).
The 2D transthoracic echocardiography revealed a severely impaired systolic function of the left ventricle, with an ejection fraction of 30\% (monoplane Simpson) and severe mitral regurgitation (Figure 2), with apical rocking and septal flash.

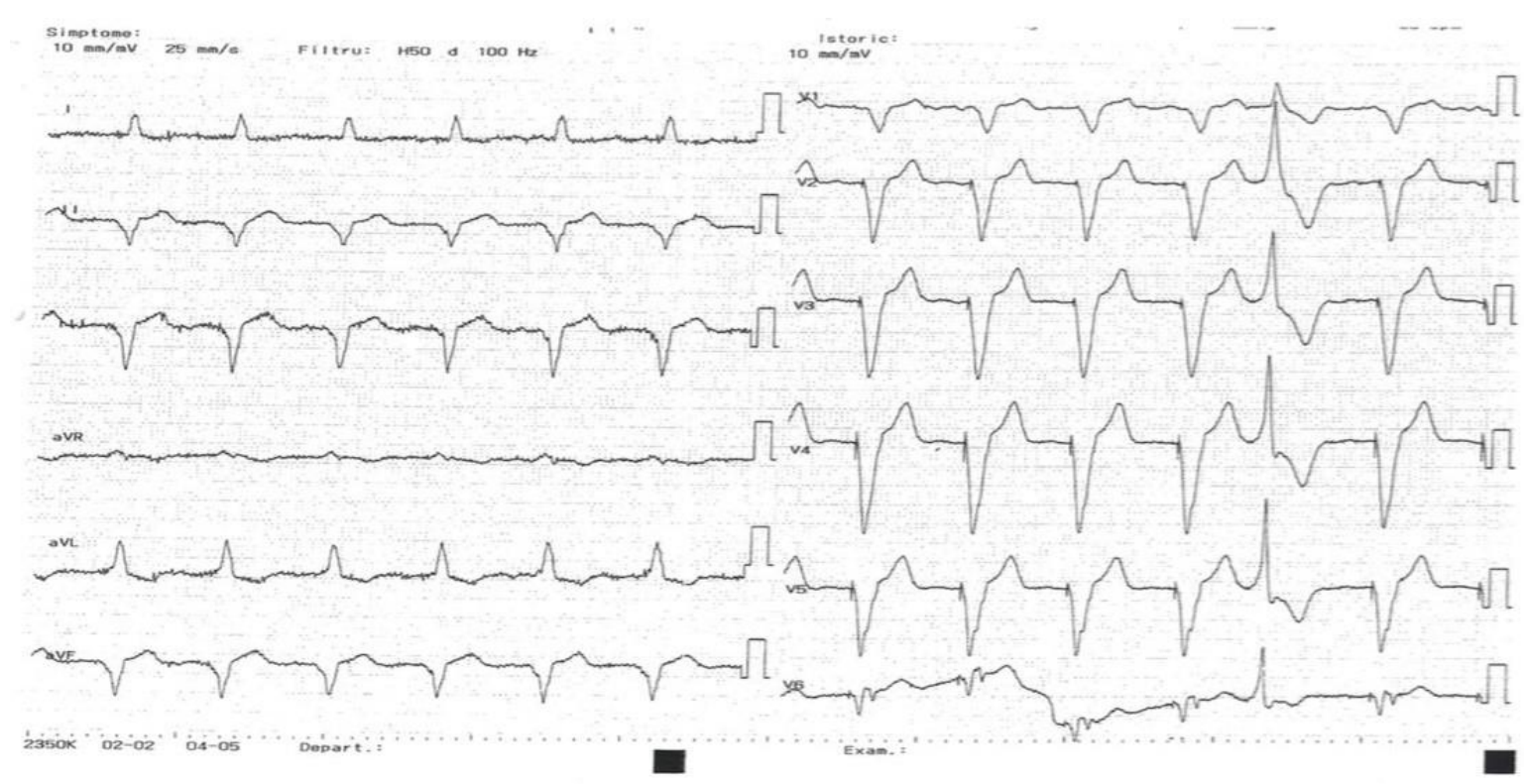

Fig. 1. 12-lead ECG with VVI 60/min pacing. QRS with left bundle branch block morphology. Isolated premature ventricular beats

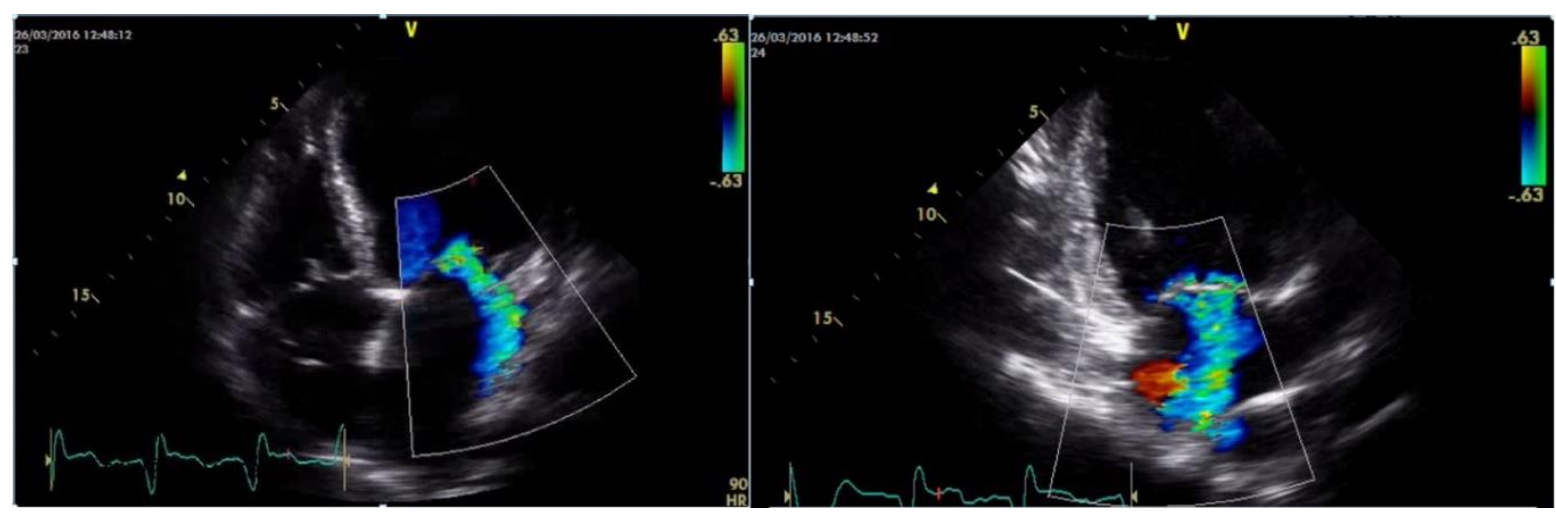

Fig. 2. 2D Transthoracic echocardiography apical 4-chamber and apical 2-chamber views during VVI pacing. Dilated left ventricle. Severe mitral regurgitation

Invasive coronary angiography was unremarkable, without coronary stenosis and patent stent. During hospitalization, the patient presented repeated episodes of asymptomatic non-sustained ventricular tachycardia (Figure $3)$. The pacemaker interrogation revealed a percentage of ventricular pacing of $76 \%$ and 1400 episodes of ventricular heart rate $(>180$ bpm). The underlying rhythm was atrial fibrillation with slow ventricular response and narrow QRS complex. Due to the discrepancies between the documented echocardiography before pacemaker implant and the current presentation, the pacemaker was set on VVI $30 \mathrm{bpm}$ and the echocardiography was repeated, revealing a mitral regurgitation significantly diminished and no left ventricular asynchrony. 


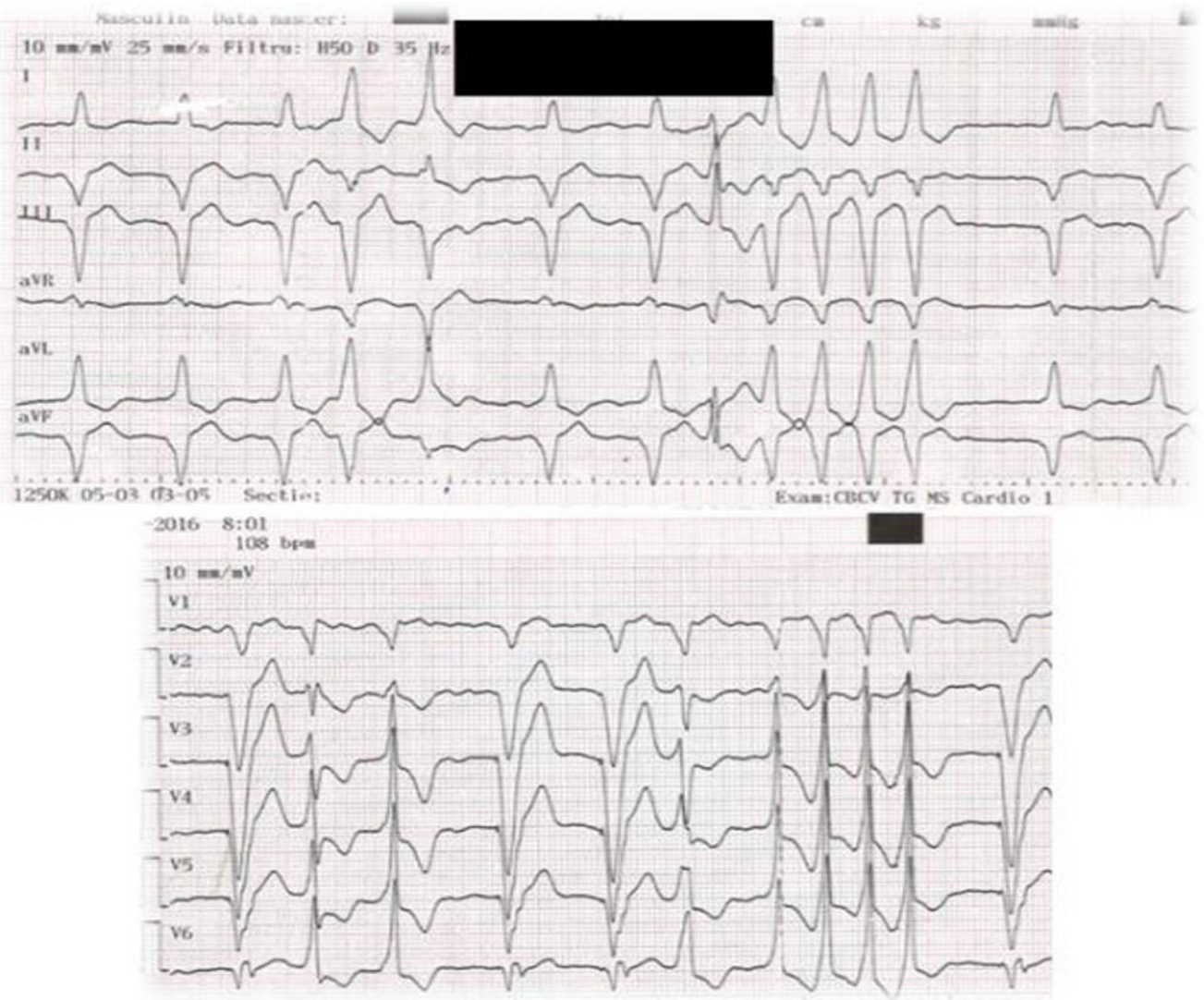

Fig. 3. 12-lead ECG. Frequent premature ventricular beats and episodes of non-sustained ventricular tachycardia originating in the basal septal area (QRS complex negative in lead II, III, aVF, transition in V2)

The medical treatment for heart failure was up-titrated at maximum tolerated doses (carvedilol $12.5 \mathrm{mg}$ b.i.d, spironolactone $25 \mathrm{mg}$ o.d., ramipril $5 \mathrm{mg}$ o.d.) and amiodarone 200 mg b.i.d. was added for ventricular arrhythmia. The pacemaker was upgraded to cardiac resynchronization therapy system with biventricular pacing (Figure 4). A left ventricular lead was implanted in a posteriorlateral vein via coronary sinus, in a distally suboptimal position due to vein caliber.
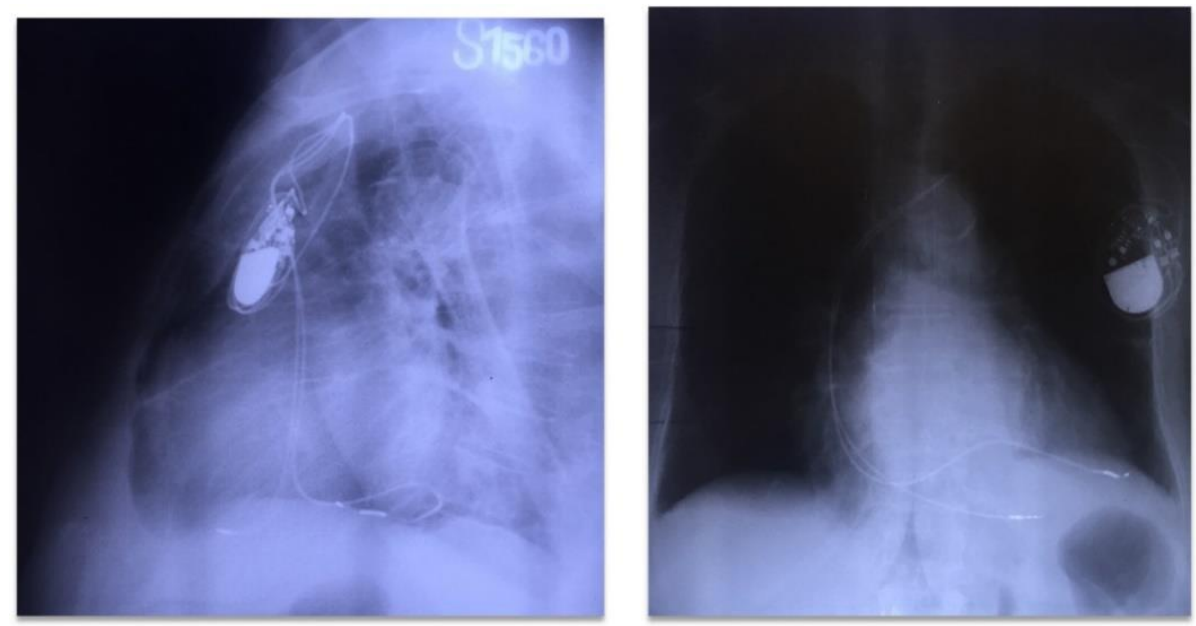

Fig. 4. Thoracic X-ray, left lateral and posterior-anterior views. Dual chamber pacemaker with a lead placed in the right ventricular apex and a lead in a posterior vein via coronary sinus 
The echocardiography post-CRT revealed an improved mitral regurgitation and left ventricular function, with an ejection fraction of
$34 \%$ and no intra or interventricular asynchrony (Figures 5 and 6).

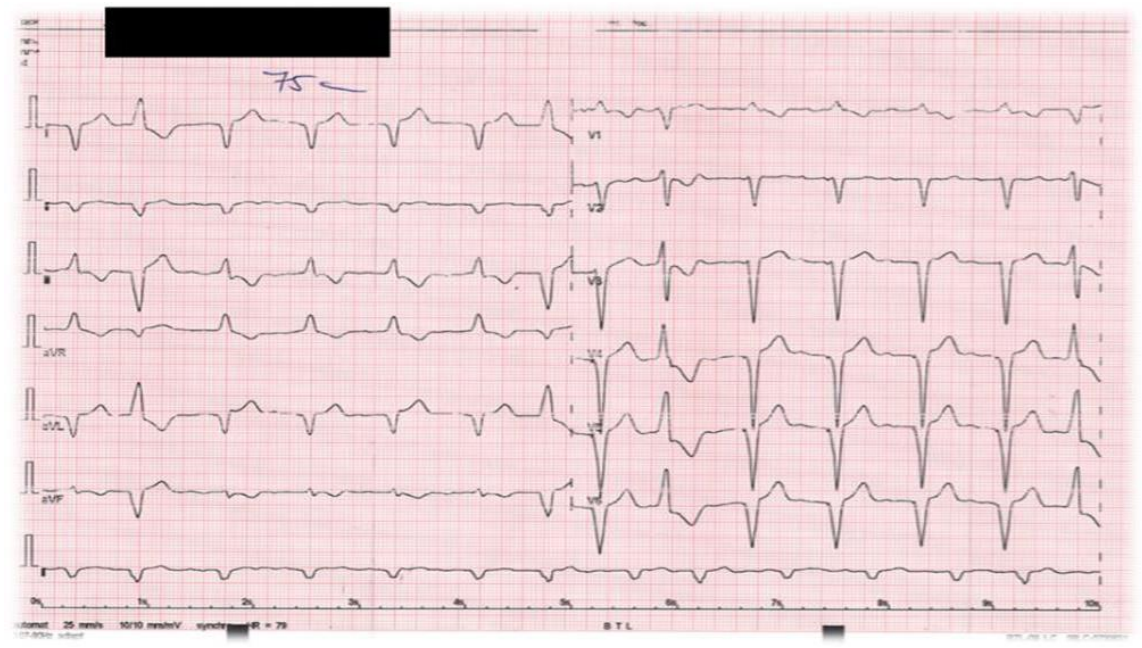

Fig. 5. 12 lead ECG after CRT

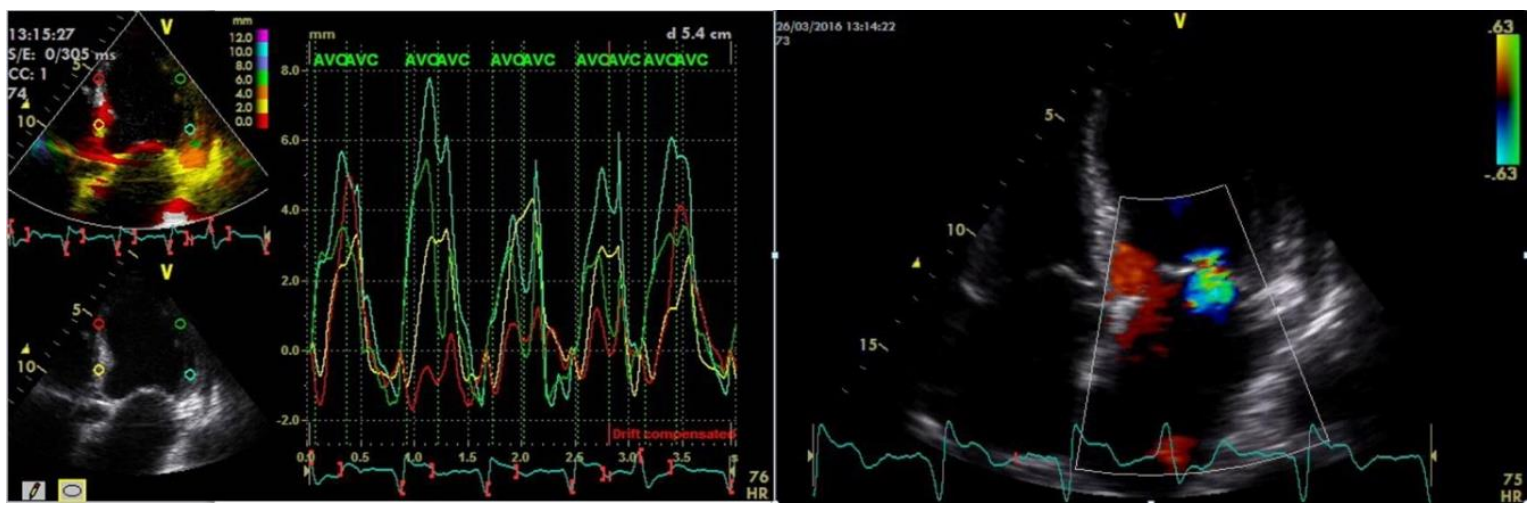

Fig. 6. Transthoracic echocardiography. Tissue Doppler in apical four-chamber view with tissue tracking (left). Apical four-chamber view, mild mitral regurgitation (right)

At discharge, the patient's symptoms were significantly improved, but the patient was lost to follow up as she moved to another country.

\section{Discussions}

We presented the case of a patient who developed heart failure secondary to right ventricular pacing, with a severely decreased left ventricular ejection fraction (from 60 to $30 \%$ in four months) and severe mitral regurgitation.

Apical right ventricular pacing induces a left bundle branch block-like electrocardiographic morphology, with a slightly different activation pattern from intrinsic left bundle branch block, with early mid-septal and late lateral wall activation [2], with the latest activation of the postero-basal left ventricular region. This leads to interventricular and left intraventricular asynchrony, and reduced cardiac output [3], causing not only worsened heart failure symptoms in patients with impaired left ventricular ejection fraction prior to pacemaker implant [4], but also new onset heart failure in approximately $20 \%$ of patients [5], particularly in patients with high percentage of ventricular pacing [6].

As a consequence of left ventricular asynchrony and dysfunction, functional mitral regurgitation is frequent in patients with right ventricular pacing, and is an independent 
prognostic factor. Its pathophysiology is complex, including mitral annulus dilatation and geometrical alteration, discrepancies in tethering and closing forces on mitral apparatus leading to impaired leaflet coaptation [7]. As expected, when our patient was not paced, the mitral regurgitation was significantely reduced, most probably due to synchronous left ventricular contraction as the instrinsic QRS complex was narrow.

Left ventricular dyssynchrony also impairs septal myocardial perfusion due to high intramyocardial pressure during early diastole [8], which might explain angina in absence of angiographic coronary arteries stenosis in our patient, with premature ventricular contractions arising in the basal septal area.

In this context, the European Society of Cardiology 2013 Guideline on cardiac pacing and resynchronization therapy recommends as a class I indication, in patients with deterioration of left ventricular systolic function and heart failure symptoms after antibradycardia pacing with a conventional pacemaker, upgrade to a cardiac

\section{References}

1. Ghani A, Delnoy PP, Ottervanger JP, Ramdat Misier AR, Smit JJ, Elvan A. Assessment of left ventricular dyssynchrony in pacing-induced left bundle branch block compared with intrinsic left bundle branch block. Europace 2011; 13(10):1504-1507.

2. Tops LF, Schalij MJ, Bax JJ. The effects of right ventricular apical pacing on ventricular function and dyssynchrony implications for therapy. J Am Coll Cardiol 2009; 54(9):764776.

3. Tops LF, Schalij MJ, Holman ER, van Erven L, van der Wall EE, Bax JJ. Right ventricular pacing can induce ventricular dyssynchrony in patients with atrial fibrillation after atrioventricular node ablation. J Am Coll Cardiol 2006; 48(8):1642-1648.

4. Freudenberger RS, Wison AC, LawrenceNelson J, Hare JM, Kostis JB, Myocardial Infarction Data Acquisition System Study Group (MIDAS 9). Permanent pacing is a risk factor for the development of heart failure. Am J Cardiol 2005; 95(5):671-674.

5. Sharma AD, Rizo-Patron $C$, Hallstrom AP, et al. Percent right ventricular pacing predicts resynchronization therapy system (CRT) [9]. Upgrade to CRT not only improves the New York Heart Association functional class, but also causes reverse remodeling of the left ventricle with improved ejection fraction, similar to patients who undergo primary CRT [10].

\section{Conclusions}

In the case we presented, a patient with atrial fibrillation which had a single chamber VVI pacemaker implanted according to current indications developed heart failure. This supports the necessity for echocardiographic follow-up in this group of patients, with early post-implant evaluation of mitral regurgitation severity as a prognostic factor for progression towards left ventricular dysfunction.

\section{Conflict of interest}

The authors declare that they have no competing interests. outcomes in the DAVID trial. Heart Rhythm 2005; 2(8):830-834.

6. Spartera M, Galderisi M, Mele D, et al. Role of cardiac dyssynchrony and resynchronization therapy in functional mitral regurgitation. Eur Heart J Cardiovasc Imaging 2016; 17(5):471480.

7. Ogano M, Iwasaki YK, Tanabe J, et al. Restoration of ventricular septal hypoperfusion by cardiac resynchronization therapy in patients with permanent right ventricular pacing. Int J Cardiol 2016; 224:353-359.

8. Brignole $M$, Auricchio A, Baron-Esquivias $G$, et al. 2013 ESC Guidelines on cardiac pacing and cardiac resynchronization therapy. Eur Heart $J$ 2013; 34(29):2281-2329.

9. Fröhlich G, Steffel J, Hürlimann D, et al. Upgrading to resynchronization therapy after chronic right ventricular pacing improves left ventricular remodelling. Eur Heart J 2010; 31(12):1477-1485.

10. Yu CM, Chan JY, Zhang Q, et al. Biventricular pacing in patients with bradycardia and normal ejection fraction. $N$ Engl $J$ Med 2009; 361(22):2123-2134. 